\title{
Oscillation Criteria for a Class of Discrete Nonlinear Fractional Equations
}

\author{
M. Reni Sagayaraj ${ }^{1}$, A.George Maria Selvam ${ }^{2}$, M.Paul Loganathan ${ }^{3}$. \\ 1,2 Sacred Heart College,Tirupattur - 635 601, S.India, \\ ${ }^{3}$ Department of Mathematics, Dravidian University, Kuppam.
}

Keywords: Difference equations, oscillation, fractional order, inequality

ABSTRACT. In this paper, we study the oscillatory behavior of the fractional difference equations of the following form

$$
\Delta\left(p(t) \Delta\left(r(t) \Delta^{\alpha} x(t)\right)^{\eta}\right)+F\left(t, \sum_{s=t_{0}}^{t-1+\alpha}(t-s-1)^{(-\alpha)} x(s)\right)=0 \text { for } t \in N_{t_{0}+1-\alpha},
$$

for $0<\alpha \leq 1, \mathrm{t}_{0} \geq \mathrm{t}>0, \Delta^{\alpha}$ denotes the Riemann-Liouville difference operator and $\eta>0$ is a quotient of odd positive integers. We establish some oscillation criteria for the equation by using Riccati transformation technique and some inequalities. An example is shown to illustrate our main results.

\section{INTRODUCTION}

The theory of fractional derivatives goes back to Leibniz's note to L'Hospital dated 30 September 1695 about the meaning of the derivative of non integer order and this led to the appearance of the theory of derivatives and integrals of arbitrary order. Fractional differential equations are generalizations of classical differential equations of integer order. In recent days, oscillatory behavior of fractional differential/difference equations has been investigated by authors, see papers [2]-[12]. Formal treatment on the subject of fractional derivatives and fractional integrals are presented in the books, see [16]-[19]. In the last few years, many authors found that fractional derivatives and fractional integrals were applied in widespread fields of science and engineering, especially in mathematical modeling real world phenomenon and simulation of systems and processes and control systems. Nowadays, many authors have investigated some qualitative aspects of fractional differential equations, such as the existence, the uniqueness and stability of solutions. But the discrete analog of fractional difference equations are studied by very few authors, see [13][15]. Now we study the oscillatory behavior of the following fractional difference equation of the form

$$
\Delta\left(p(t) \Delta\left(r(t) \Delta^{\alpha} x(t)\right)^{\eta}\right)+F\left(t, \sum_{s=t_{0}}^{t-1+\alpha}(t-s-1)^{(-\alpha)} x(s)\right)=0 \text { for } t \in N_{t_{0}+1-\alpha},
$$

$\mathrm{t} \geq \mathrm{t}>0, \eta$ is the ratio of two odd positive integers and $\alpha \Delta$ denotes the Riemann-Liouville difference operator of order $0<\alpha \leq 1$.

$\left(\mathrm{H}_{1}\right) p(t), r(t)$ are positive sequences $\sum_{s=t_{0}}^{\infty} \frac{1}{p(s)}=\infty, \sum_{s=t_{0}}^{\infty} \frac{1}{r(s)}=\infty$, and $f: R \rightarrow R$ is a continuous function such that $f(x) /\left(x^{n}\right) \geq k$ for a certain constant $k>0$ and for all $x \neq 0$.

$\left(\mathrm{H}_{2}\right) \quad F(t, G) \in C\left(\left[t_{0}, \infty\right) \times R ; R\right)$ and there exists a real sequence $q(t)$ such that $F(t, G) / G^{\eta} \geq q(t)$ for $\mathrm{G} \neq 0$ and $x \neq 0, t \geq t_{0}$. 
A solution $x(t)$ of (1) is said to be oscillatory if it is neither eventually positive nor eventually negative; otherwise, it is nonoscillatory. Equation (1) is said to be oscillatory if all its solutions are oscillatory.

\section{Preliminaries and Basic Lemmas}

In this section, we provide preliminary results of discrete fractional calculus, which will be used throughout this paper.

Definition 2.1. (see [13]) Let $v>0$. The $v$-th fractional sum of $\mathrm{f}$ is defined by

$$
\Delta^{-v} f(t)=\frac{1}{\Gamma(v)} \sum_{s=a}^{t-v}(t-s-1)^{(v-1)} f(s)
$$

where $\mathrm{f}$ is defined for $\mathrm{s} \equiv a \bmod (1)$ and $\Delta^{-v}$ is defined for $\mathrm{t} \equiv(\mathrm{a}+\mathrm{v}) \bmod (1)$ and $\mathrm{t}^{(v)}=\frac{\Gamma(t+1)}{\Gamma(t-v+1)}$ The fractional sum $\Delta^{-v} f$ maps functions defined on $\mathrm{N}_{a}$ to functions defined on $\mathrm{N}_{a+v}$.

Definition 2.2. (see [13]) Let $\mu>0$ and $\mathrm{m}-1<\mu<\mathrm{m}$, where $\mathrm{m}$ denotes a positive integer, $\mathrm{m}=\lceil\mu\rceil$. Set $\nu=m-\mu$. The $\mu$-th fractional Riemann-Liouville difference is defined as

$$
\Delta^{\mu} f(t)=\Delta^{m-v} f(t)=\Delta^{m} \Delta^{-v} f(t)
$$

In order to discuss our results in Section 3, now we state the following lemma [1].

Lemma 2.3. Let A be a positive real number, B a positive arbitrary number and let $\gamma$ be a quotient of odd positive integers. Then

$$
B u-A u^{\gamma+1 / \lambda} \leq \frac{\gamma^{\gamma}}{(\gamma+1)^{\gamma+1}} \frac{B^{\gamma+1}}{A^{\gamma}}
$$

Lemma 3.1. Let $x(t)$ be a solution of (1) and let

$$
G(t)=\sum_{s=t_{0}}^{t-1+\alpha}(t-s-1)^{(-\alpha)} x(s)
$$

then

$$
\Delta(G(t))=\Gamma(1-\alpha) \Delta^{\alpha}(x(t)) .
$$

$$
\begin{aligned}
G(t)=\sum_{s=t_{0}}^{t-1+\alpha}(t-s-1)^{(-\alpha)} x(s) & =\sum_{s=t_{0}}^{t-1+\alpha}(t-s-1)^{(1-\alpha)-1} x(s) \\
& =\Gamma(1-\alpha) \Delta^{-(1-\alpha)} x(t)
\end{aligned}
$$

which implies

$$
\Delta(G(t))=\Gamma(1-\alpha) \Delta \Delta^{-(1-\alpha)} x(t)=\Gamma(1-\alpha) \Delta^{\alpha} x(t) .
$$

Lemma 3.2. Assume that $x(t)$ is an eventually positive solution of equation (1). If $x(t)>0, \Delta^{\alpha} x(t)>0$, and $G(t)>0$, then $\Delta\left(r(t) \Delta^{\alpha} x(t)\right)^{\eta}>0$ for $t \geq t_{0}$. 
Proof. Let $x(t)$ be an eventually positive solution of (1) for $t_{1} \geq t_{0}$. From (1), $\Delta\left(p(t) \Delta\left(r(t) \Delta^{\alpha} x(t)\right)^{\eta}\right) \leq 0$ for $t \geq t_{1}$. We claim that there is a $t_{2} \geq t_{1}$ such that $\Delta\left(r(t) \Delta^{\alpha} x(t)\right)^{\eta}>0$. for $t \geq t_{2}$. Suppose to the contrary that $\Delta\left(r(t) \Delta^{\alpha} x(t)\right)^{\eta} \leq 0$ for $t \geq t_{2}$. Since $p(t)>0$ and $p(t) \Delta\left(r(t) \Delta^{\alpha} x(t)\right)^{\eta}$ is non increasing, there exists a negative constant $\delta$ and $t_{3} \geq t_{2}$ such that $p(t) \Delta\left(r(t) \Delta^{\alpha} x(t)\right)^{\eta} \leq \delta$ for $t \geq t_{3}$. Dividing by $p(t)$ and summing from $t_{3}$ to $t-1$, we obtain

$$
\left(r(t) \Delta^{\alpha} x(t)\right)^{\eta} \leq\left(r\left(t_{3}\right) \Delta^{\alpha} x\left(t_{3}\right)\right)^{\eta}+\sum_{s=t_{3}}^{t-1} \frac{\delta}{p(s)} .
$$

Letting $\mathrm{t} \rightarrow \infty$, we obtain $\mathrm{x}(\mathrm{t}) \alpha \Delta \rightarrow-\infty$ which is a contradiction, since $\Delta^{\alpha} \mathrm{x}(\mathrm{t})>0$. This completes the proof.

Lemma 3.3. Assume that $x(t)$ is an eventually positive solution of equation (1) and suppose that Lemma (3.2) holds. Then there exists $t_{1}>t_{0}$ such that $\Delta^{\alpha} x(t)>0, \Delta\left(r(t) \Delta^{\alpha} x(t)\right)^{\eta}>0$ Then we have

$$
(\Delta G(t))^{\eta} \geq \Gamma(1-\alpha)^{\eta} \Delta\left(r(t) \Delta^{\alpha} x(t)\right)^{\eta} \frac{1}{r(t)^{\eta}} \sum_{s=t_{1}}^{t-1} \frac{1}{p(s)} .
$$

Proof. From equation (1), we see that $\Delta\left(p(t) \Delta\left(r(t) \Delta^{\alpha} x(t)\right)^{\eta}\right) \leq 0$ and hence $\left(r(t) \Delta^{\alpha} x(t)\right)^{\eta}$ is non increasing. Then we obtain

$$
\begin{aligned}
&\left(r(t) \Delta^{\alpha} x(t)\right)^{\eta}=\left(r\left(t_{1}\right) \Delta^{\alpha} x\left(t_{1}\right)\right)^{\eta}+\sum_{s=t_{1}}^{t-1} \frac{p(s) \Delta\left(r(s) \Delta^{\alpha} x(t)\right)^{\eta}}{p(s)} \\
& \geq p(t) \Delta\left(r(t) \Delta^{\alpha} x(t)\right)^{\eta} \sum_{s=t_{1}}^{t-1} \frac{1}{p(s)} \\
&(\Delta G(t))^{\eta} \geq \Gamma(1-\alpha)^{\eta} p(t) \Delta\left(r(t) \Delta^{\alpha} x(t)\right)^{\eta} \frac{1}{r(t)^{\eta}} \sum_{s=t_{1}}^{t-1} \frac{1}{p(s)} .
\end{aligned}
$$

Theorem 3.4. Suppose that conditions $\mathrm{H}_{1}$ and $\mathrm{H}_{2}$ hold. If there exists a positive sequence $\rho(\mathrm{s})$ such that

$$
\limsup _{t \rightarrow \infty} \sum_{s=t_{0}}^{t-1}\left(\rho(s) q(s)-\frac{\left(\Delta \rho_{+} s\right)^{2}}{4 \rho^{2}(s+1) R(s)}\right)=\infty
$$

Where

$$
\Delta \rho_{+}(s)=\max (\rho(s), 0) \text { and } R(t)=\frac{2^{1-\eta} \rho(t) \Gamma(1-\alpha)^{\eta} \frac{1}{r(t)^{\eta}} \sum_{s=t_{0}}^{t-1} \frac{1}{p(s)}}{\rho^{2}(t+1) p(t)} .
$$

Then every solution of equation (1) is oscillatory.

Proof. Suppose to the contrary that equation (1) has a nonoscillatory solution $x(t)$. Without loss of generality, we may assume that $x(t)>0$ on $1 \mathrm{t} \geq \mathrm{t}$. We define the function $\mathrm{w}(\mathrm{t})$ by Riccati substitution 


$$
w(t)=\rho(t) \frac{p(t) \Delta\left(r(t) \Delta^{\alpha} x(t)\right)^{\eta}}{G^{\eta}(t)} \text { for } t \geq t_{1} .
$$

Then we have $w(t)>0$ for $t \geq t_{1}$. Also, we have

$$
\begin{aligned}
\Delta w(t) & =\Delta \rho(t) \frac{w(t+1)}{\rho(t+1)} \\
& +\frac{\rho(t) \Delta\left(p(t) \Delta\left(r(t) \Delta^{\alpha} x(t)\right)^{\eta}\right) G^{\eta}(t+1)-\rho(t) p(t+1) \Delta\left(r(t+1) \Delta^{\alpha} x(t+1)\right)^{\eta} \Delta G^{\eta}(t)}{G^{\eta}(t+1) G^{\eta}(t)} \\
& \leq \Delta \rho_{+}(t) \frac{w(t+1)}{\rho(t+1)}-\rho(t) q(t)-\frac{\rho(t) p(t+1) \Delta\left(r(t+1) \Delta^{\alpha} x(t+1)\right)^{\eta} \Delta G^{\eta}(t)}{G^{2 \eta}(t+1)}
\end{aligned}
$$

Now using the inequality (see [1])

$$
x^{\beta}-y^{\beta} \geq 2^{1-\beta}(x-y)^{\beta} \text {, for all } x \geq y>0 \text { and } \beta \geq 1
$$

we have

$$
p(t) \Delta\left(r(t) \Delta^{\alpha} x(t)\right)^{\eta} \geq p(t+1) \Delta\left(r(t+1) \Delta^{\alpha} x(t+1)\right)^{\eta}
$$

Using the above inequality, we obtain

$$
\begin{aligned}
& \Delta w(t) \leq \Delta \rho_{+}(t) \frac{w(t+1)}{\rho(t+1)}-\rho(t) q(t)-\frac{2^{1-\eta} \rho(t) p(t+1) \Delta\left(r(t+1) \Delta^{\alpha} x(t+1)\right)^{\eta}(\Delta G(t))^{\eta}}{G^{2 \eta}(t+1)} \\
& \leq \Delta \rho_{+}(t) \frac{w(t+1)}{\rho(t+1)}-\rho(t) q(t)-\frac{2^{1-\eta} \rho(t) \Gamma(1-\alpha)^{\eta} \Delta\left(r(t) \Delta^{\alpha} x(t)\right)^{\eta} \frac{1}{r(t)^{\eta}} \sum_{s=t_{1}}^{t-1} \frac{1}{p(s)}}{\frac{\rho^{2}(t+1) p(t+1) \Delta\left(r(t+1) \Delta^{\alpha} x(t+1)\right)^{\eta}}{w(t+1)^{2}}} \\
& \Delta w(t) \leq \Delta \rho_{+}(t) \frac{w(t+1)}{\rho(t+1)}-\rho(t) q(t)-\frac{2^{1-\eta} \Gamma(1-\alpha)^{\eta} \rho(t) \frac{p(t+1)}{p(t)} \Delta\left(r(t+1) \Delta^{\alpha} x(t+1)\right)^{\eta} \frac{1}{r(t)^{\eta}} \sum_{s=t_{1}}^{t-1} \frac{1}{p(s)}}{\frac{\rho^{2}(t+1) p(t+1) \Delta\left(r(t+1) \Delta^{\alpha} x(t+1)\right)^{\eta}}{w(t+1)^{2}}} \\
& \leq \Delta \rho_{+}(t) \frac{w(t+1)}{\rho(t+1)}-\rho(t) q(t)-\frac{2^{1-\eta} \rho(t) \Gamma(1-\alpha)^{\eta} \frac{1}{r(t)^{\eta}} \sum_{s=t_{1}}^{t-1} \frac{1}{p(s)}}{\rho^{2}(t+1) p(t)} w(t+1)^{2} \\
& =\Delta \rho_{+}(t) \frac{w(t+1)}{\rho(t+1)}-\rho(t) q(t)-R(t) w(t+1)^{2} \\
& R(t)=\frac{2^{1-\eta} \rho(t) \Gamma(1-\alpha)^{\eta} \frac{1}{r(t)^{\eta}} \sum_{s=t_{1}}^{t-1} \frac{1}{p(s)}}{\rho^{2}(t+1) p(t)} \\
& A=R(t), \quad B=\frac{\Delta \rho_{+}(t)}{\rho(t+1)}, \text { and } u=w(t+1) \text {. }
\end{aligned}
$$


and using Lemma (2.3) with $\gamma=1$, we obtain

$$
\begin{aligned}
B u-A U^{2} & \leq \frac{1}{2^{2}} \frac{B^{2}}{A} \\
& =\frac{1}{4} \frac{\left(\frac{\Delta \rho_{+}(t)}{\rho(t+1)}\right)^{2}}{R(t)} \\
& =\frac{\left(\Delta \rho_{+}(t)\right)^{2}}{4 \rho^{2}(t+1) R(t)}
\end{aligned}
$$

From (5), we conclude that

$$
\Delta w(t) \leq-\rho(t) q(t)+\frac{\left(\Delta \rho_{+}(t)\right)^{2}}{4 \rho^{2}(t+1) R(t)} .
$$

Summing the above inequality from $\mathrm{t}_{1}$ of $\mathrm{t}-1$ we have

$$
\sum_{s=t_{1}}^{t-1}\left(\rho(s) q(s)-\frac{\left(\Delta \rho_{+}(s)\right)^{2}}{4 \rho^{2}(s+1) R(s)}\right) \leq w\left(t_{1}\right)-w(t) \leq w\left(t_{1}\right)<\infty, \text { for } t \geq t_{1}
$$

Letting $t \rightarrow \infty$, we get

$$
\limsup _{t \rightarrow \infty} \sum_{s=t_{1}}^{t-1}\left(\rho(s) q(s)-\frac{\left(\Delta \rho_{+}(s)\right)^{2}}{4 \rho^{2}(s+1) R(s)}\right) \leq w\left(t_{1}\right)<\infty
$$

which contradicts (3). The proof is complete.

Theorem 3.5. Suppose that conditions $\left(\mathrm{H}_{1}\right)$ and $\left(\mathrm{H}_{2}\right)$ hold. Furthermore, assume that there exists a positive sequence $\rho(\mathrm{t})$ such that

If

$$
\begin{aligned}
& H(t, t)=0 \text { for } t \geq 0 H(t, s)>0 \quad t>s \geq 0 \\
& \Delta_{2} H(t, s)=H(t, s+1)-H(t, s) \leq 0 \text { for } t \geq s \geq 0 .
\end{aligned}
$$

solution of (1) is oscillatory.

$$
\limsup _{t \rightarrow \infty} \frac{1}{H\left(t, t_{0}\right)} \sum_{s=t_{0}}^{t-1}\left(\rho(s) q(s) H(t, s)-\frac{h_{+}^{2}(t, s)}{4 H(t, s) R(s)}\right)=\infty
$$

$$
\text { Where } h_{+}(t, s)=\Delta_{2} H(t, s)+\frac{H(t, s) \Delta \rho_{+}(s)}{\rho(s+1)} \text { and } \Delta \rho_{+}(s)=\max [\Delta \rho(s), 0] \text {. }
$$

Then every

Proof. Suppose the contrary that $\mathrm{x}(\mathrm{t})$ is a nonoscillatory solution of (1). Without loss of generality, we may assume that $x(t)$ is an eventually positive solution of (1). We proceed as in the proof of Theorem (3.4) to get (10). Multiplying (10) by $H(t, s)$ and summing from $\mathrm{t}_{1}$ to $\mathrm{t}-1$, we obtain

$$
\begin{aligned}
& \sum_{s=t_{1}}^{t-1} \rho(s) q(s) H(t, s) \leq-\sum_{s=t_{1}}^{t-1} H(t, s) \Delta w(s)+\sum_{s=t_{1}}^{t-1} H(t, s) \Delta \rho_{+}(s) \frac{w(s+1)}{b(s+1)} \\
& -\sum_{s=t_{1}}^{t-1} H(t, s) R(s) w^{2}(s+1)
\end{aligned}
$$

Using summation by parts formula, we get 


$$
\begin{aligned}
-\sum_{s=t_{1}}^{t-1} H(t, s) \Delta w(s) & =-[H(t, s) w(s)]_{s=t_{1}}^{t}+\sum_{s=t_{1}}^{t-1} w(s+1) \Delta_{2} H(t, s) \\
& =H\left(t, t_{1}\right) w\left(t_{1}\right)+\sum_{s=t_{1}}^{t-1} w(s+1) \Delta_{2} H(t, s)
\end{aligned}
$$

where $\Delta_{2} H(t, s)=H(t, s+1)-H(t, s)$. For $t \geq t_{1}$ we have

$$
\begin{gathered}
\sum_{s=t_{1}}^{t-1} \rho(s) q(s) H(t, s) \leq H\left(t, t_{1}\right) w\left(t_{1}\right)+\sum_{s=t_{1}}^{t-1} w(s+1) \Delta_{2} H(t, s)+\sum_{s=t_{1}}^{t-1} H(t, s) \Delta \rho_{+}(s) \frac{w(s+1)}{\rho(s+1)}-\sum_{s=t_{1}}^{t-1} H(t, s) R(s) w^{2}(s+1) \\
\leq H\left(t, t_{1}\right) w\left(t_{1}\right)+\sum_{s=t_{1}}^{t-1}\left(\Delta_{2} H(t, s)+\frac{H(t, s) \Delta \rho_{+}(s)}{b(s+1)}\right) w(s+1)-\sum_{s=t_{1}}^{t-1} H(t, s) R(s) w^{2}(s+1) \\
\leq H\left(t, t_{1}\right) w\left(t_{1}\right)+\sum_{s=t_{1}}^{t-1}\left(h_{+}(t, s) w(s+1)-H(t, s) R(s) w^{2}(s+1)\right)
\end{gathered}
$$

where

$$
h_{+}(t, s)=\Delta_{2} H(t, s)+\frac{H(t, s) \Delta \rho_{+}(s)}{\rho(s+1)}
$$

Taking

$A=R(t) H(t, s), \quad B=h_{+}(t, s)$, and $u=w(t+1)$ and using Lemma 2.3 with $\gamma=1$, we get

$$
\begin{aligned}
B u-A U^{2} & \leq \frac{1}{2^{2}} \frac{B^{2}}{A} \\
& =\frac{1}{4} \frac{h_{+}^{2}(t, s)}{H(t, s) R(t)}
\end{aligned}
$$

We have $\Delta_{2} H(t, s) \leq 0$ for $t>s \geq t_{0}, 0<H\left(t, t_{1}\right) \leq H\left(t, t_{0}\right)$ for $t>t_{1} \geq t_{0}$.

$$
\begin{aligned}
\sum_{s=t_{1}}^{t-1} \rho(s) q(s) H(t, s) \leq H\left(t, t_{1}\right) w\left(t_{1}\right)+\sum_{s=t_{1}}^{t-1} & \frac{h_{+}^{2}(t, s)}{4 H(t, s) R(s)} \\
\sum_{s=t_{1}}^{t-1}\left(\rho(s) q(s) H(t, s)-\frac{h_{+}^{2}(t, s)}{4 H(t, s) R(s)}\right) & \leq H\left(t, t_{1}\right) w\left(t_{1}\right) \\
& \leq H\left(t, t_{0}\right) w\left(t_{1}\right) .
\end{aligned}
$$

Since, $0<H(t, s) \leq H\left(t, t_{0}\right)$ for $t>s \geq t_{0}$, we have $0<\frac{H(t, s)}{H\left(t, t_{0}\right)} \leq 1$ for $t>s \geq t_{0}$.

Hence it follows that

$$
\begin{aligned}
\frac{1}{H\left(t, t_{0}\right)} \sum_{s=t_{0}}^{t_{1}-1}\left(\rho(s) q(s) H(t, s)-\frac{h_{+}^{2}(t, s)}{4 H(t, s) R(s)}\right)= & \frac{1}{H\left(t, t_{0}\right)} \sum_{s=t_{0}}^{t_{1}-1}\left(\rho(s) q(s) H(t, s)-\frac{h_{+}^{2}(t, s)}{4 H(t, s) R(s)}\right) \\
& +\frac{1}{H\left(t, t_{0}\right)} \sum_{s=t_{1}}^{t-1}\left(\rho(s) q(s) H(t, s)-\frac{h_{+}^{2}(t, s)}{4 H(t, s) R(s)}\right) \\
\leq & \frac{1}{H\left(t, t_{0}\right)} \sum_{s=t_{0}}^{t_{1}-1} \rho(s) q(s) H(t, s)+w\left(t_{1}\right) \\
\leq & \sum_{s=t_{0}}^{t_{1}-1} \rho(s) q(s)+w\left(t_{1}\right) .
\end{aligned}
$$


Letting $\mathrm{t} \rightarrow \infty$, we have

$$
\limsup _{t \rightarrow \infty} \frac{1}{H\left(t, t_{0}\right)} \sum_{s=t_{0}}^{t-1}\left(\rho(s) q(s) H(t, s)-\frac{h_{+}^{2}(t, s)}{4 H(t, s) R(s)}\right) \leq \sum_{s=t_{0}}^{t-1} \rho(s) q(s)+w\left(t_{1}\right)<\infty,
$$

which is a contradiction to (11). The proof is complete.

Example 3.6. Consider the following fractional difference equation

$$
\Delta\left(t \Delta\left(t^{1 / 3} \Delta^{\alpha}(x(t))\right)^{3}\right)+t^{2}\left(\sum_{s=0}^{t-1+\alpha}(t-s-1)^{(-\alpha)} x(s)\right)^{3}=0
$$

Here, $\alpha=\frac{1}{2}, \eta=3, p(t)=t, q(t)=t^{2}$ and $r(t)=t^{1 / 3}$

We will apply Theorem (3.4) and it remains show that condition (6) is satisfied. Taking $\rho(\mathrm{s})=\mathrm{s}$, we obtain

$$
\limsup _{t \rightarrow \infty} \sum_{s=t_{0}}^{t-1}\left(\rho(s) q(s)-\frac{\left(\Delta \rho_{+}(s)\right)^{2}}{4 \rho^{2}(s+1) R(s)}\right)=\lim _{t \rightarrow \infty} \sup \sum_{s=t_{0}}^{t-1} s\left(s^{2}-\frac{1}{\pi^{3 / 2} \sum_{s=t_{0}}^{\infty} \frac{1}{s}}\right)=\infty
$$

which implies that (6) holds. Therefore, by Theorem (3.4) every solution of (15) is oscillatory.

\section{References}

[1] G.H. Hardy, J.E. Littlewood, G. Polya, Inequalities, Cambridge University Press, Cambridge (1959).

[2] Said R. Grace, Ravi P. Agarwal, Patricia J.Y. Wong, Agacik Zafer, On the oscillation of fractional differential equations, FCAA, Vol15, No.2(2012).

[3] Da-Xue Chen, Oscillation criteria of fractional differential equations, Advances in Difference Equations 2012, 2012:33.

[4] Da-Xue Chen, Oscillatory behavior of a class of fractional differential equations with damping, U.P.B. Sci. Bull., Series A, Vol. 75, Iss. 1, 2013.

[5] Da-Xue Chen, Pei-Xin Qu, Yong-Hong Lan, Forced oscillation of certain fractional differential equations, Advances in Di_erence Equations 2013, 2013:125.

[6] Run Xu, Oscillation Criteria for Nonlinear Fractional Differential Equations, Hindawi Publishing Corporation, Journal of Applied Mathematics, Volume 2013, Article ID 971357, 7 pages, http://dx.doi.org/10.1155/2013/971357.

[7] Yizhuo Wang, Zhenlai Han, Ping Zhao and Shurong Sun, On the oscillation and asymptotic behavior for a kind of fractional differential equations. Advances in Difference Equations 2014, 2014:50

[8] S.Lourdu Marian, M. Reni Sagayaraj, A.George Maria Selvam, M.Paul Loganathan, Oscillation of fractional nonlinear difference equations, Mathematica Aeterna, Vol. 2, 2012, no. 9, 805 - 813.

[9] S. Lourdu Marian, M. Reni Sagayaraj, A. George Maria Selvam, M. Paul Loganathan, Oscillation of Caputo Like Discrete Fractional Equations, International Journal of Pure and Applied Mathematics, Volume 89 No. 5 2013, 667-677. 
[10] M. Reni Sagayaraj, A. George Maria Selvam and M. Paul Loganathan, Oscillation of a Class of Forced Fractional Difference Equations, International J. of Math. Sci. \& Engg. Appls. (IJMSEA), ISSN 0973-9424, Vol. 8 No. I (January, 2014), pp. 349-356

[11] M. Reni Sagayaraj, A.George Maria Selvam, M.Paul Loganathan, On the oscillation nonlinear fractional nonlinear di_erence equations, Mathematica Aeterna, Vol. 4, 2014, no. 1, 91 - 99.

[12] Zhenlai Han, Yige Zhao, Ying Sun, Chao Zhang, Oscillation for a class of fractional differential equation, Hindawi Publishing Corporation, Discrete Dynamics in Nature and Society, Volume 2013, Article ID 390282, 6 pages.

[13] Fulai Chen, Xiannan Luo, Y. Zhou, Existence results for nonlinear fractional difference equations, Advances in Difference Equations, Volume 2011, Article ID 713201, 12 pages.

[14] F. M. Atici, P. W. Eloe, Initial value problems in discrete fractional calculus, Proceedings of the American Mathematical Society, Vol. 137, No. 3, pp. 981-989, 2009.

[15] G.A. Anastassiou, Discrete fractional calculus and inequalities, http://arxiv.org/abs/ 0911.3370v1.

[16] K. S. Miller and B. Ross, An Introduction to the Fractional Calculus and Fractional Differential Equations, JohnWiley \& Sons, New York, NY, USA, 1993.

[17] K. Diethelm, The Analysis of Fractional Differential Equations, Springer, Berlin, 2010.

[18] A.A. Kilbas, H.M. Srivastava, J.J. Trujillo, Theory and Applications of Fractional Differential Equations. North-Holland Math. Studies 204, Elsevier, Amsterdam, 2006.

[19] I. Podlubny, Fractional Differential Equations, Academic Press, San Diego, Calif, USA, 1999. 